\title{
Needs Assessment In Patients Surgically Treated For Head And Neck Cancer - A Randomized Controlled Trial
}

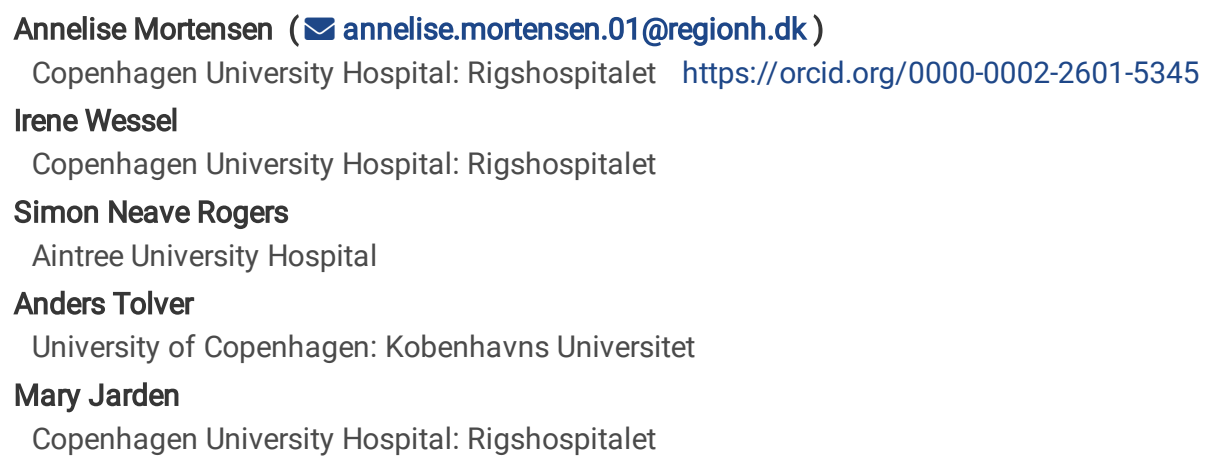

\section{Research Article}

Keywords: head and neck cancer, needs assessment, quality of life, rehabilitation, symptom, burden

Posted Date: August 12th, 2021

DOI: https://doi.org/10.21203/rs.3.rs-682615/v1

License: (c) (i) This work is licensed under a Creative Commons Attribution 4.0 International License. Read Full License

Version of Record: A version of this preprint was published at Supportive Care in Cancer on January 27th, 2022. See the published version at https://doi.org/10.1007/s00520-021-06759-9. 


\section{Abstract}

\section{Purpose:}

Investigate whether a head and neck cancer specific needs assessment tool integrated in nursing rehabilitation consultations early in the postsurgical period would improve quality of life and symptom burden in patients surgically treated. Further, to assess the feasibility of carrying out needs assessments during consultations.

\section{Methods:}

92 surgically treated head and neck cancer patients at the Department of Otolaryngology, Head and Neck Surgery and Audiology, Copenhagen University Hospital, Denmark were enrolled.

A two-arm randomized controlled trial design was used. Both arms received nursing rehabilitation consultations before discharge, approximately two weeks and two months post-operative. Patients in the intervention group had their needs assessed using an assessment tool.

Primary outcome was quality of life. Secondary outcomes were symptom burden and referrals for multi-disciplinary rehabilitation follow-up.

\section{Results:}

No significant differences were found in quality of life or symptom burden. However, notable more patients in the intervention group were referred for rehabilitation.

\section{Conclusion:}

The intervention suggests that important needs were identified and addressed, especially emotional and existential needs, which were accommodated through referrals and professional advice. Nursing rehabilitation consultations using a needs assessment instrument may ensure that patient preferences and priorities are incorporated in their care.

\section{Trial Registration}

ClinicalTrials.com (NCT03443258). Date of registration: May 31 ${ }^{\text {st }}, 2018$

\section{Introduction}

Patients who have undergone surgery for head and neck cancer (HNC) experience considerable adverse effects and significant symptom burden [1]. Surgical treatment alters functional anatomy [2], such that patients with HNC experience dysfunction across physical, functional, emotional, social, and existential domains [3]. Dysfunction can result in unmet needs being difficult for patients to express and for health care professionals (HCP) to identify [4]. A high proportion of patients with HNC have unmet needs [5], with some studies showing up to 60\% [6], and if left unmanaged, may negatively impact quality of life (QoL), emotional wellbeing, and daily living [6]. Needs may present early or late in a patient's trajectory [1, 5]. Identification and intervention regarding these concerns can potentially reduce symptom burden and lead to improvement in health-related Quality of Life (HRQoL) [6].

Assessment of patient needs related to symptom management and rehabilitation is recommended after treatment to mitigate early and late detrimental effects [7]. The Danish Health Authority recommends assessing cancer patient needs early in the post-treatment phase, including physical, social, psychological, and existential needs [8]. The assessment may use national needs assessment instruments recommended by the Regions of Denmark [8]; however, these are generic and do not cater to specific needs of patients with HNC [9].

Head and neck nurse specialists play a key role in assessing patient needs. Advice, information, and support in managing physical symptoms and emotional reactions are recommended to be integrated in nursing consultations $[11,12]$. Wider multidisciplinary team involvement is shown to have positive impact on patient HRQoL [12].

A variety of instruments are available to help identify the needs of patients with HNC [14-16]. Some instruments are based on HRQoL questionnaires linking poor outcomes or anxiety and depression to unmet needs [16]. Others are based on symptom burden questionnaires [17]. However, patient-reported outcome (PRO) measures developed for research purposes often measure HRQoL and may not be useful in identifying patient needs [3]. A systematic review and content comparison of self-report measures of unmet needs used in patients with HNC favoured the Patient Concerns Inventory (PCI) compared to 13 other tools [18]. PCl is a 56-item prompt list used in clinics to facilitate patients raising concerns that might otherwise be missed [19].

We hypothesized that an HNC needs assessment tool integrated in nursing rehabilitation consultations early in the post-surgical period would improve HRQoL and symptom burden. The aim was to investigate the effect of the HNC needs assessment tool on HRQoL, symptom burden and 
types and numbers of follow-up referrals. Further, to assess the feasibility of carrying out needs' assessments during nursing rehabilitation consultations.

\section{Methods}

\section{Sample and Settings}

Designed as a two-arm randomized controlled trial (RCT) with a control group (CG) and an intervention group (IG), the study recruited patients from Department of Otolaryngology, Head and Neck Surgery and Audiology, Copenhagen University Hospital, Denmark (Dept. H\&N), between June 1, 2018 and August 31, 2019. The inclusion criteria were: >18 years of age, either newly diagnosed or recurrent and operated for HNC within the last few days; able to speak and understand Danish. The exclusion criteria were: treated surgically for thyroid or parotic cancers, referred to adjuvant radio/chemotherapy, and diagnosed with unstable psychiatric illness.

Patients were randomized via Research Electronic Data Capture (REDCap), an electronic system for research data containing a randomization module [20]. Patients were randomly assigned 1:1 to either CG or IG, and stratified according to medical status; newly diagnosed or recurrence, and ASA classification [21]. Block randomization was applied. Assignments were not blinded to either investigator or patients, but the statistician was blinded to treatment allocation.

\section{Study Context}

The study was conducted at Dept. H\&N, a tertiary, tax-funded, public health care facility with an uptake area of 2.6 million people. Post-operative assessment consultations are provided for all patients prior to discharge [8], while rehabilitation takes place in the primary sector [22]. In Denmark, nurse rehabilitation consultations include a formal, systematic needs assessment for symptom management and rehabilitation, but some aspects are done collaboratively with swallowing therapists and surgeons. Follow-up in the primary sector is multidisciplinary and may include referral to or advice about contacting, a speech pathologist, swallowing therapist, physiotherapist, psychologist or counsellor.

\section{Procedures}

CG received usual post-operative care, including three rehabilitation consultations by a nurse on the rehabilitation team, which did not include a needs assessment tool or follow any specific flow. Consultations took place immediately after the appointment with the surgeon, where the nurse had accompanied the patient. The nurse followed up on the surgeon's consultation and assessed the patient's needs based on hospital records and systematic questioning. Recommendations, referrals, and advice for follow-up at a hospital or in primary sector were decided upon and patients were handed a selection of leaflets, based on the nurses' professional perspective.

Consultations were offered prior to discharge (time-point 1), 7-10 days after discharge (time-point 2), and two months post-surgery (time-point 3).

IG received usual post-operative care, including three nursing rehabilitation consultations taking place at the same time-points as CG and conducted by primary investigator AM or a project nurse. The consultations applied PCI [23] as a needs assessment instrument, where patients select items they would like to discuss with the nurse related to functional, emotional, social, and existential areas. In addition to encouraging patients to express their needs, concerns, and symptoms, they can choose items they find important and meaningful to discuss. To promote integration of needs assessment and facilitate patient involvement in the consultations [24,25], these followed a certain sequence inspired by Smith's patient-centered interviewing [26]. Patients were informed about the purpose of the consultation at the outset and received guidance on completing the $\mathrm{PCl}$ on iPad. Nurse and patients briefly discussed results and identified needs or concerns patients wished to discuss with the surgeon. The nurse accompanied the patient to the surgeon's consultation and ensured chosen concerns were discussed. After the consultation with the surgeon, the nurse followed up on this and remaining needs and concerns based on the PCl were discussed. Any needs nurses deemed important from a professional perspective were added to the discussion. Finally, collaboratively with the patient, recommendations, referrals, and advice for follow-up at hospital or in primary sector was decided upon guided by a management manual.

Prior to commencement of the study, a management manual for discussing and acting on PCl items was developed, as well as a leaflet informing the patient on how to access support in the primary sector, e.g. psychologist, counsellor, smoking and alcohol cessation programs, chaplain, or other religious support. Further, a Danish linguistic validation of PCl was conducted, and an IT solution on iPad developed, easily allowing an overview of items. The Region's Centre for IT, Medical Technology and Telephony Services approved the solution.

\section{Outcome Measurements}

Primary outcome was HRQoL on European Organisation for Research and Treatment of Cancer Quality of Life Questionnaire (EORTC QLQ), and symptoms using EORTC QLQ-Head and Neck 35 (H\&N35)[27]. The questionnaires have 35 items comprising a global QoL and global health status (GHS) scale, five functional and nine symptom scales on EORTC QIQ and 18 symptom scales the last five of which are binary on H\&N35, with scores ranging from 0-100. HRQoL was measured using the GHS/QoL scale which consists of two items rating the overall health and overall QoL. When measuring changes over time, it is suggested changes above 10 points is considered clinically relevant [28]. EORTC QLQ-H\&N35 was 
completed at two time-points: baseline (before randomization) and seven days after the two-month post-treatment appointment, either electronically via email generated by REDCap or letter by regular mail. Patients rated scores based on past seven days.

The secondary outcomes included symptom prevalence and severity evaluated by MD Anderson Symptom Inventory-Head and Neck (MDASI-HN) module [29], a multi-symptom PRO measure for clinical and research use. The MDASI-HN module comprises a 28-item questionnaire measuring symptoms and their severity in patients with HNC using a numerical rating scale from $0-10$, wherein 0 means a symptom is not present and 10 means as bad as imaginable. Scores between 1-4 are considered mild; 5-6 moderate, and 7-10 severe [30]. When measuring changes over time, a minimum important difference is 0.98-1.21 [29]. MDASI-HN was completed at the same time-points as EORTC QIQ-H\&N35. Patients rated scores based on past 24 hours.

The types and number of multidisciplinary referrals were registered at three time-points: before discharge, 7-10 days and two months posttreatment. Demographic, medical data and comorbidity using the Charlson Comorbidity Index [31] were registered at baseline. The outcomes and items chosen on the $\mathrm{PCl}$ were registered for IG.

\section{Statistical Analysis}

Sample size was based on results from studies with similar groups of patients reporting a standard deviation (SD) of size 20 on within-group changes of GHS/QoL on EORTC QLQ [32]. A between-group difference of 15 for changes from baseline to post-intervention was considered clinically relevant. Based on two-sample t-test, we found 29 patients in each group were required to obtain a power of 0.80 . To account for an expected dropout of $20 \%$, we decided to include at least 72 patients. Descriptive statistics was used to describe demographics and baseline characteristics of the two groups. For numerical variables, we reported mean and range, and for categorical variables, numbers and percentages. For EORTC QLQ-H\&N35 and MDASI-HN we presented mean, SD, and number of data available at each assessment time for both groups. We used a linear mixed model with treatment, assessment time, and their interaction as fixed effects, and subject as random effect for estimation of withingroup changes and for between-group comparison of changes. The Wald test was used to test the hypothesis that within-group changes or between-group differences equal zero. For binary outcomes from H\&N35, we reported estimated prevalence and standard error for each combination of assessment time and treatment group. McNemar's test was used to test for within-group changes over time of paired binary outcomes. P-values < 0.05 were reported as statistically significant, but they must be interpreted with care. Due to many secondary outcomes, risk of reporting false positive results is high; hence, this should be regarded as an exploratory analysis. Types and number of referrals were reported for each group at each assessment time. For IG, PCI items chosen were calculated at each assessment time and reported. All statistical analyses were carried out using R [33].

\section{Results}

Of 244 eligible patients, 92 (38\%) were included. Sixty-four patients were excluded for not meeting inclusion criteria, 32 were unreachable for inclusion, and $56(23 \%)$ declined to participate. Patients were randomly allocated to CG ( $\mathrm{n}=48), 13$ of whom withdrew or were excluded, and IG (n =44), 14 of whom withdrew or were excluded (Fig. 1).

Groups were comparable, except for tumour sites, where CG had nine patients with tumours in larynx (23\%) and eight in pharynx (21\%), compared to IG, with five in larynx (16\%) and two in pharynx (6\%) (Table 1). 
Table 1

Baseline demographic and medical characteristics

Intervention $(n=44)$

Control $(n=48)$

Gender

Women

$12(27 \%)$

$16(33 \%)$

Men

$32(73 \%)$

$32(67 \%)$

Age, years

$64.4(37-80)$

$64.9(45-85)$

Smoking status

Never smoker

$8(18 \%)$

$10(21 \%)$

Previous smoker

$28(64 \%)$

$29(60 \%)$

Previous smoker package-years

$35.7(1-76.5)$

41.9 (5-153)

Smoker

$8(18 \%)$

$9(19 \%)$

Smoker package-years

$59.2(8,4-112)$

$56.8(36,8-90)$

Alcohol

No alcohol

$7(16 \%)$

$2(4 \%)$

Less than 7 units/wk.

$13(30 \%)$

$17(35 \%)$

7-14 units/wk.

$12(27 \%)$

$17(35 \%)$

More than 14 units/wk.

$12(27 \%)$

$12(25 \%)$

BMI

$26.7(18.3-38.1)$

$24.1(17-33.4)$

Partner

Living alone

$30(68 \%)$

33 (69\%)

Cohabiting

$13(30 \%)$

$15(31 \%)$

Employment status

Retired/early retirement

$24(55 \%)$

$27(56 \%)$

Years since last employment

Min: 1 Max: 43

Min: 0 Max: 24

Mean: 13.4

Mean: 8.8

Employed (part-time)

$7(16 \%)$

$3(6 \%)$

Employed (full-time)

$12(27 \%)$

$15(31 \%)$

Rehabilitation benefits

$1(2 \%)$

$1(2 \%)$

Social security

0

$2(4 \%)$

Educational level, years

Lower, 7-9

$5(11 \%)$

$7(15 \%)$

Middle, 12-15

$32(72 \%)$

$31(65 \%)$

Higher, 17-20

$7(16 \%)$

$10(21 \%)$

Diagnosis

$\mathrm{n}=32$

$\mathrm{n}=39$

Larynx

$5(16 \%)$

$9(23 \%)$

\section{Legend:}

Package years $=$ No. of cigarettes daily $\mathrm{x}$ no of years $/ 20 ;$ Units $=1$ bottle beer, 1 glass wine, 1 glass dessert wine; 1 glass spirits;

PFO $=$ able to carry out all normal activity without restriction, PF1 = restricted in strenuous activity but ambulatory and able to carry out light work, PF2 = ambulatory and capable of all self-care but unable to carry out any work activities; up and about more than $50 \%$ of waking hours, PF3 = symptomatic and in a chair or in bed for greater than $50 \%$ of the day but not bedridden 


\begin{tabular}{|c|c|c|}
\hline & Intervention $(n=44)$ & Control $(n=48)$ \\
\hline Pharynx (total) & $2(6 \%)$ & $8(21 \%)$ \\
\hline Oral cavity & $25(78 \%)$ & $22(56 \%)$ \\
\hline Tumour stage & $\mathrm{n}=32$ & $\mathrm{n}=39$ \\
\hline Stage I & $12(38 \%)$ & $1949 \%)$ \\
\hline Stage II & $7(22 \%)$ & $4(10 \%)$ \\
\hline Stage III & $10(31 \%)$ & $5(13 \%)$ \\
\hline Stage IV & $1(3 \%)$ & $5(13 \%)$ \\
\hline Missing & $2(6 \%)$ & $6(15 \%)$ \\
\hline Newly diagnosed & $34(77 \%)$ & $36(75 \%)$ \\
\hline Recurrences & $10(23 \%)$ & $12(25 \%)$ \\
\hline \multicolumn{3}{|c|}{ Comorbidities number (Charlson) } \\
\hline 0 & $20(45 \%)$ & $2756 \%)$ \\
\hline 1 & $11(25 \%)$ & $1123 \%)$ \\
\hline 2 & $7(16 \%)$ & $8(17 \%)$ \\
\hline 3 & $3(7 \%)$ & $1(2 \%)$ \\
\hline 4 & $3(7 \%)$ & $1(2 \%)$ \\
\hline \multicolumn{3}{|c|}{ Performance Status (PF) } \\
\hline PF 0 & $7(16 \%)$ & $12(25 \%)$ \\
\hline PF 1 & $28(64 \%)$ & $3267 \%)$ \\
\hline PF 2 & $9(20 \%)$ & $3(6 \%)$ \\
\hline PF 3 & 0 & $1(2 \%)$ \\
\hline \multicolumn{3}{|l|}{ Legend: } \\
\hline \multicolumn{3}{|c|}{ Package years $=$ No. of cigarettes daily $\times$ no of years $/ 20$; Units $=1$ bottle beer, 1 glass wine, 1 glass dessert wine; 1 glass spirits; } \\
\hline $\begin{array}{l}\text { PF0 = able to carry } \\
\text { work, PF2 = ambul } \\
\text { PF3 = symptomati }\end{array}$ & $\begin{array}{l}=\text { restricted in strenuo } \\
\text { carry out any work a } \\
\text { of the day but not bed }\end{array}$ & $\begin{array}{l}\text { y and able to carry out light } \\
\text { ore than } 50 \% \text { of waking hours, }\end{array}$ \\
\hline
\end{tabular}

\section{Primary Outcome}

Primary outcome on EORTC QLQ, showed no statistically significant difference between within-group change on GHS/QoL: 3.8 (95\% Cl-10.7-18.4; $p=0.60)$. However, both groups showed statistically significant improvements over time (IG: $P=0.01, C G: P=0.04)$. For EORTC QLQ, there were significant improvements in both groups for emotional functioning (CG: $P<0.01$, IG: $P<0.01$ ); for symptom scales pain (CG: $P=0.01, I G: P<0.01$ ) and appetite loss (CG: $P=0.03, \mathrm{IG}: \mathrm{P}=0.05$ ). Symptom scales for nausea/vomiting, dyspnoea, constipation, diarrhoea, and financial situation all showed mean scores below 20 (bottom fifth of the scale) with non-significant improvements over time in both groups. Symptom burden changed for one or both groups for following scales or items on H\&N35: decreased pain (CG: $P=0.01$, IG: $P<0.01$ ); improved problems with senses (CG: $P<$ 0.01 ); improved problems with speech (CG: $P=0.02)$; decreased felt ill (IG: $P=0.02)$; decreased use of painkillers (CG: $P=0.03, I G: P<0.01$ )

(Table 2).

Table 2. European Organisation for Research and Treatment of Cancer Quality of Life Questionnaire (EORTC QLQ) and Head and Neck 35 (H\&N35)

\section{EORTC QLQ}




\begin{tabular}{|c|c|c|c|c|c|c|c|c|c|c|}
\hline & \multicolumn{2}{|c|}{ Baseline } & \multicolumn{2}{|c|}{ Post } & \multicolumn{6}{|c|}{ Linear mixed model } \\
\hline & $\mathrm{n}$ & $\begin{array}{l}\text { Mean } \\
\text { (SD) }\end{array}$ & $\mathrm{n}$ & Mean (SD) & $\begin{array}{l}\text { Change } \\
\text { within }\end{array}$ & $95 \% \mathrm{Cl}$ & $\begin{array}{l}\mathrm{P}- \\
\text { value }\end{array}$ & $\begin{array}{l}\text { Change } \\
\text { between }\end{array}$ & $95 \% \mathrm{Cl}$ & P-value \\
\hline \multicolumn{11}{|c|}{ Global health status } \\
\hline Control & 46 & $59.4(25.0)$ & 29 & $70.1(23.5)$ & 10.7 & $0.6-20.7$ & 0.04 & & & \\
\hline Intervention & 41 & $57.1(26.3)$ & 27 & $72.2(25.2)$ & 14.5 & 4.0- 25.0 & 0.01 & 3.8 & $-10.7-18.4$ & 0.60 \\
\hline \multicolumn{11}{|c|}{ Functional scales } \\
\hline \multicolumn{11}{|c|}{ Physical functioning } \\
\hline Control & 48 & $83.0(17.1)$ & 29 & 85.7 (16.9) & 2.4 & $-3.6-8.3$ & 0.43 & & & \\
\hline Intervention & 42 & $80.0(23.5)$ & 27 & 86.7 (20.9) & 4.7 & $-1.5-11.0$ & 0.13 & 2.4 & $-6.2-11.0$ & 0.59 \\
\hline \multicolumn{11}{|c|}{ Role functioning } \\
\hline Control & 48 & $81.6(29.2)$ & 29 & $80.5(24.8)$ & -1.7 & $-11.9-8.4$ & 0.73 & & & \\
\hline Intervention & 41 & $78.5(30.3)$ & 27 & $79.6(31.8)$ & 2.6 & $-8.1-13.3$ & 0.63 & 4.3 & $\begin{array}{l}-10.4- \\
19.0\end{array}$ & 0.56 \\
\hline \multicolumn{11}{|c|}{ Emotional functioning } \\
\hline Control & 46 & $70.8(19.6)$ & 29 & $81.9(20.8)$ & 11.9 & $3.9-19.9$ & $<0.01$ & & & \\
\hline Intervention & 41 & $67.8(26.8)$ & 27 & $80.6(27.0)$ & 13.6 & $5.2-21.9$ & $<0.01$ & 1.6 & $-9.9-13.2$ & 0.78 \\
\hline \multicolumn{11}{|c|}{ Cognitive functioning } \\
\hline Control & 47 & $86.2(18.2)$ & 29 & $89.1(18.0)$ & 4.1 & $-2.2-10.4$ & 0.19 & & & \\
\hline Intervention & 41 & $75.6(28.2)$ & 27 & $82.7(26.3)$ & 5.8 & $-0.9-12.4$ & 0.09 & 1.6 & $-7.5-10.8$ & 0.72 \\
\hline \multicolumn{11}{|c|}{ Social functioning } \\
\hline Control & 47 & $87.9(21.1)$ & 29 & $88.5(17.9)$ & 1.0 & $-6.9-8.9$ & 0.80 & & & \\
\hline Intervention & 40 & $89.2(19.4)$ & 27 & $87.0(24.2)$ & -1.8 & $-10.1-6.6$ & 0.68 & -2.8 & $-14.3-8.7$ & 0.63 \\
\hline \multicolumn{11}{|c|}{ Symptom scales } \\
\hline \multicolumn{11}{|l|}{ Fatigue } \\
\hline Control & 48 & $27.9(27.7)$ & 29 & $24.5(22.9)$ & -4.9 & $-13.8-3.9$ & 0.27 & & & \\
\hline Intervention & 43 & $31.9(27.2)$ & 27 & $25.1(23.6)$ & -6.4 & $-15.6-2.9$ & 0.17 & -1.4 & $\begin{array}{l}-14.2- \\
11.4\end{array}$ & 0.83 \\
\hline \multicolumn{11}{|c|}{ Nausea and vomiting } \\
\hline Control & 48 & $3.1(8.9)$ & 29 & $1.1(4.3)$ & -1.3 & $-5.8-3.3$ & 0.57 & & & \\
\hline Intervention & 43 & 7.4 (17.6) & 27 & $7.4(14.9)$ & 0.3 & $-4.4-5.0$ & 0.89 & 1.6 & $-4.9-8.1$ & 0.62 \\
\hline \multicolumn{11}{|l|}{ Pain } \\
\hline Control & 48 & $26.0(26.4)$ & 29 & $8.6(13.1)$ & -17.5 & $\begin{array}{l}-26.9- \\
(-8.1)\end{array}$ & $<0.01$ & & & \\
\hline Intervention & 43 & $23.6(24.7)$ & 27 & $10.5(17.4)$ & -12.3 & $\begin{array}{l}-22.2- \\
(-2.5)\end{array}$ & 0.01 & 5.2 & $-8.4-18.8$ & 0.45 \\
\hline \multicolumn{11}{|l|}{ Dyspnoea } \\
\hline Control & 46 & $13.8(23.9)$ & 29 & $14.9(22.9)$ & 1.9 & $-6.3-10.1$ & 0.65 & & & \\
\hline Intervention & 39 & $17.1(27.4)$ & 27 & $11.1(22.6)$ & -8.3 & $-16.9-0.3$ & 0.06 & -10.2 & $-22.1-1.7$ & 0.09 \\
\hline \multicolumn{11}{|l|}{ Insomnia } \\
\hline Control & 47 & $30.5(30.2)$ & 29 & $24.1(26.6)$ & -8.5 & $-19.2-2.3$ & 0.12 & & & \\
\hline Intervention & 42 & 34.1 (33.3) & 27 & $25.9(33.8)$ & -9.7 & $-20.9-1.5$ & 0.09 & -1.3 & $\begin{array}{l}-16.8- \\
14.3\end{array}$ & 0.87 \\
\hline
\end{tabular}




\begin{tabular}{|c|c|c|c|c|c|c|c|c|c|c|}
\hline Control & 48 & $22.2(33.2)$ & 29 & $10.3(20.1)$ & -11.1 & $\begin{array}{l}-21.1- \\
(-1.0)\end{array}$ & 0.03 & & & \\
\hline Intervention & 43 & $22.5(31.5)$ & 27 & $\begin{array}{l}8.6 \\
(23.7)\end{array}$ & -10.4 & $-20.9-0.0$ & 0.05 & 0.6 & $\begin{array}{l}-13.8- \\
15.1\end{array}$ & 0.93 \\
\hline \multicolumn{11}{|l|}{ Constipation } \\
\hline Control & 48 & $11.8(26.2)$ & 29 & $9.2(19.7)$ & -2.8 & $-12.9-7.2$ & 0.57 & & & \\
\hline Intervention & 42 & $15.1(27.7)$ & 27 & $7.4(16.9)$ & -7.0 & $-17.5-3.6$ & 0.19 & -4.1 & $\begin{array}{l}-18.7- \\
10.4\end{array}$ & 0.57 \\
\hline \multicolumn{11}{|l|}{ Diarrhoea } \\
\hline Control & 47 & $10.6(19.8)$ & 29 & $11.5(24.0)$ & 0.5 & $-7.5-8.5$ & 0.90 & & & \\
\hline Intervention & 40 & $10.8(23.1)$ & 27 & $\begin{array}{l}7.4 \\
(16.9)\end{array}$ & -4.6 & $-13.0-3.9$ & 0.28 & -5.1 & $-16.7-6.5$ & 0.38 \\
\hline \multicolumn{11}{|c|}{ Financial situation } \\
\hline Control & 47 & $5.7(18.8)$ & 27 & $9.9(18.1)$ & 3.1 & $-4.4-10.7$ & 0.41 & & & \\
\hline Intervention & 41 & $12.2(26.6)$ & 27 & 12.3 (29.5) & 0.0 & $-7.7-7.7$ & 1.00 & -3.1 & $-13.9-7.7$ & 0.56 \\
\hline
\end{tabular}

\section{H\&N35}




\begin{tabular}{|c|c|c|c|c|c|c|c|c|c|c|}
\hline & \multicolumn{2}{|c|}{ Baseline } & \multicolumn{2}{|c|}{ Post } & \multicolumn{6}{|c|}{ Linear mixed model } \\
\hline & $\mathbf{n}$ & Mean (SD) & $\mathrm{n}$ & Mean (SD) & Change within & $95 \% \mathrm{Cl}$ & $\begin{array}{l}\mathrm{P} \text { - } \\
\text { value }\end{array}$ & $\begin{array}{l}\text { Change } \\
\text { between }\end{array}$ & $95 \% \mathrm{Cl}$ & P-value \\
\hline \multicolumn{11}{|l|}{ Pain } \\
\hline Control & 46 & $21.6(21.4)$ & 29 & $10.8(13.3)$ & -10.2 & $-18.1--2.3$ & 0.01 & & & \\
\hline Intervention & 42 & $32.5(28.3)$ & 26 & $11.6(15.3)$ & -19.1 & $-27.4--10.9$ & $<0.01$ & -8.9 & $-20.4-2.5$ & 0.12 \\
\hline \multicolumn{11}{|l|}{ Swallowing } \\
\hline Control & 47 & $13.7(22.7)$ & 29 & $10.1(18.0)$ & -2.7 & $-10.8-5.4$ & 0.51 & & & \\
\hline Intervention & 42 & $15.6(25.7)$ & 26 & $8.7(16.7)$ & -5.9 & $-14.5-2.6$ & 0.17 & -3.2 & $-15.1-8.6$ & 0.58 \\
\hline \multicolumn{11}{|l|}{ Senses } \\
\hline Control & 47 & $9.6(18.6)$ & 29 & $26.4(32.6)$ & 16.7 & $7.9-25.5$ & $<0.01$ & & & \\
\hline Intervention & 40 & $17.1(30.0)$ & 26 & $16.0(25.6)$ & -0.9 & $-10.3-8.5$ & 0.85 & -17.6 & $-30.5-(-4.7)$ & 0.01 \\
\hline \multicolumn{11}{|l|}{ Speech } \\
\hline Control & 44 & $13.4(19.0)$ & 29 & $24.1(26.4)$ & 9.1 & $1.4-16.7$ & 0.02 & & & \\
\hline Intervention & 39 & $13.8(19.5)$ & 26 & $12.8(20.3)$ & -1.0 & $-9.1-7.2$ & 0.81 & -10.0 & $-21.2-1.2$ & 0.08 \\
\hline \multicolumn{11}{|l|}{ Social eating } \\
\hline Control & 44 & $14.8(26.8)$ & 29 & $10.6(20.5)$ & -3.3 & $-12.5-5.9$ & 0.48 & & & \\
\hline Intervention & 38 & $14.8(21.2)$ & 26 & $11.3(26.4)$ & -1.2 & $-11.2-8.7$ & 0.81 & 2.1 & $-11.5-15.6$ & 0.76 \\
\hline \multicolumn{11}{|c|}{ Social contact } \\
\hline Control & 45 & $3.8(8.6)$ & 29 & $5.7(11.1)$ & 2.3 & $-3.5-8.1$ & 0.43 & & & \\
\hline Intervention & 39 & $7.1(15.4)$ & 26 & $6.1(17.5)$ & -0.5 & $-6.8-5.7$ & 0.86 & -2.8 & $-11.4-5.7$ & 0.51 \\
\hline \multicolumn{11}{|c|}{ Less sexuality } \\
\hline Control & 39 & $33.3(40.5)$ & 27 & $38.3(36.9)$ & -1.4 & $-15.4-12.6$ & 0.84 & & & \\
\hline Intervention & 29 & $19.0(33.5)$ & 23 & $16.7(34.1)$ & 1.8 & $-14.0-17.6$ & 0.82 & 3.2 & $-17.9-24.4$ & 0.76 \\
\hline \multicolumn{11}{|l|}{ Teeth } \\
\hline Control & 44 & $5.3(12.3)$ & 29 & $6.9(16.4)$ & 2.1 & $-5.3-9.5$ & 0.58 & & & \\
\hline Intervention & 38 & $14.0(32.5)$ & 25 & $5.3(18.5)$ & -2.8 & $-10.8-5.2$ & 0.49 & -4.9 & $-15.7-6.0$ & 0.37 \\
\hline \multicolumn{11}{|c|}{ Mouth opening } \\
\hline Control & 47 & $7.1(16.9)$ & 29 & $10.3(20.1)$ & 3.3 & $-7.1-13.6$ & 0.53 & & & \\
\hline Intervention & 42 & $11.9(28.3)$ & 26 & $6.4(21.1)$ & -5.5 & $-16.4-5.4$ & 0.32 & -8.7 & $-23.8-6.3$ & 0.25 \\
\hline \multicolumn{11}{|l|}{ Dry Mouth } \\
\hline Control & 47 & $22.0(28.9)$ & 29 & $18.4(27.6)$ & -4.3 & $-16.7-8.2$ & 0.50 & & & \\
\hline Intervention & 40 & 36.7 (38.3) & 26 & $26.9(26.7)$ & -9.0 & $.-22.3-4.3$ & 0.18 & -4.8 & $-23.0-13.5$ & 0.60 \\
\hline \multicolumn{11}{|l|}{ Sticky saliva } \\
\hline Control & 45 & $16.3(26.2)$ & 29 & $19.5(22.7)$ & 4.1 & $-7.0-15.3$ & 0.46 & & & \\
\hline Intervention & 39 & $20.5(28.2)$ & 26 & $16.7(28.7)$ & -3.3 & $-15.1-8.6$ & 0.58 & -7.4 & $-23.7-9.0$ & 0.37 \\
\hline \multicolumn{11}{|l|}{ Coughing } \\
\hline Control & 45 & $28.9(29.0)$ & 29 & $18.4(19.1)$ & -9.4 & $-18.6-(-0.2)$ & 0.05 & & & \\
\hline Intervention & 41 & $22.0(24.3)$ & 26 & $24.4(29.1)$ & 1.9 & $-7.8-11.6$ & 0.70 & 11.3 & $-2.1-24.6$ & 0.10 \\
\hline
\end{tabular}




\begin{tabular}{|c|c|c|c|c|c|c|c|c|c|c|}
\hline Control & 46 & $15.9(26.0)$ & 28 & $9.5(17.8)$ & -6.2 & $-17.0-4.6$ & 0.25 & & & \\
\hline Intervention & 39 & $19.7(27.3)$ & 25 & $5.5(12.5)$ & -14.4 & $-25.9-(-2.8)$ & 0.02 & -8.2 & $-24.0-7.7$ & 0.30 \\
\hline Binary items & $n$ & Mean (SE) & $\mathrm{n}$ & Mean (SE) & Change within & & $\begin{array}{l}\text { P- } \\
\text { value }\end{array}$ & & & \\
\hline \multicolumn{11}{|l|}{ Pain killers } \\
\hline Control & 45 & $51.1(7.5)$ & 29 & $24.1(7.1)$ & & & 0.03 & & & \\
\hline Intervention & 36 & $66.7(7.9)$ & 26 & $19.2(7.7)$ & & & $<0.01$ & & & \\
\hline \multicolumn{11}{|c|}{ Nutritional supplements } \\
\hline Control & 43 & $21.9(6.2)$ & 29 & $27.6(8.3)$ & & & 1.00 & & & \\
\hline Intervention & 36 & $8.3(4.6)$ & 25 & $24.0(8.5)$ & & & 0.37 & & & \\
\hline \multicolumn{11}{|l|}{ Feeding tube } \\
\hline Control & 46 & $8.7(4.2)$ & 29 & $3.4(3.4)$ & & & 0.48 & & & \\
\hline Intervention & 35 & $5.7(3.9)$ & 26 & $3.8(3.7)$ & & & 1.00 & & & \\
\hline \multicolumn{11}{|l|}{ Weight loss } \\
\hline Control & 45 & $33.3(7.0)$ & 29 & $13.8(6.4)$ & & & 0.11 & & & \\
\hline Intervention & 37 & $24.3(7.1)$ & 26 & $15.4(7.1)$ & & & 1.00 & & & \\
\hline \multicolumn{11}{|l|}{ Weight gain } \\
\hline Control & 46 & $6.5(3.6)$ & 29 & $37.9(9.0)$ & & & 0.03 & & & \\
\hline Intervention & 36 & $8.3(4.6)$ & 25 & $28.0(9.0)$ & & & 0.13 & & & \\
\hline
\end{tabular}

Mean scores and SD are based on raw data. Change scores and $\mathrm{Cl}$ are based on linear mixed model. For last five binary items the table displays estimated prevalence (in \%) and corresponding standard error instead of mean and SD. Within-group changes are based on McNemar's test

The response rate to EORTC QLQ-H\&N35 post-time dropped by 6 (17 \%) for CG and 3 (10\%) for IG.

\section{Secondary Outcomes}

Changes in symptom prevalence and severity measured by MDASI-HN did not show significant differences between groups. Overall range for mean scores of core symptoms was $0.2-4.7$, with highest scores in fatigue; disturbed sleep; being distressed; drowsy/sleepy; dry mouth and sadness. Overall range for mean scores of head and neck-specific symptoms was $0.3-4.9$, with highest scores in problems with mucus in mouth/throat; difficulty with swallowing/chewing; and difficulty with voice/speech. Significant improvements over time were observed in many areas in both groups. Overall range of mean scores for interference symptoms was 1.0-5.3, with highest scores in activity, mood, and work, with slight improvements in both groups over time (Table 3). The response rate to MDASI-HN dropped by $2(6 \%)$ in CG and 2 (7\%) for IG at post-treatment assessment.

\section{Table 3. MD Anderson Symptom Inventory-Head \& Neck}




\begin{tabular}{|c|c|c|c|c|c|c|c|c|c|c|}
\hline & \multicolumn{2}{|c|}{ Baseline } & \multicolumn{2}{|c|}{ Post } & \multicolumn{6}{|c|}{ Linear mixed model } \\
\hline & $\mathrm{n}$ & $\begin{array}{l}\text { Mean } \\
\text { (SD) }\end{array}$ & $\mathrm{n}$ & $\begin{array}{l}\text { Mean } \\
\text { (SD) }\end{array}$ & $\begin{array}{l}\text { Change } \\
\text { within }\end{array}$ & $95 \% \mathrm{Cl}$ & P-value & $\begin{array}{l}\text { Change } \\
\text { between }\end{array}$ & $95 \% \mathrm{Cl}$ & P-value \\
\hline \multicolumn{11}{|c|}{ Core symptom } \\
\hline \multicolumn{11}{|l|}{ Pain } \\
\hline Control & 46 & $\begin{array}{l}4.7 \\
(3.1)\end{array}$ & 33 & $\begin{array}{l}1.1 \\
(2.2)\end{array}$ & -3.6 & $-4.8--2.4$ & 0.00 & & & \\
\hline Intervention & 41 & $\begin{array}{l}4.6 \\
(2.7)\end{array}$ & 28 & $\begin{array}{l}1.7 \\
(2.7)\end{array}$ & -2.9 & $-4.2--1.6$ & 0.00 & 0.7 & $-1.0-2.4$ & 0.38 \\
\hline \multicolumn{11}{|l|}{ Fatigue } \\
\hline Control & 47 & $\begin{array}{l}4.7 \\
(3.4)\end{array}$ & 30 & $\begin{array}{l}2.5 \\
(3.0)\end{array}$ & -2.2 & $-3.2--1.2$ & 0.00 & & & \\
\hline Intervention & 39 & $\begin{array}{l}4.7 \\
(2.6)\end{array}$ & 27 & $\begin{array}{l}1.9 \\
(2.5)\end{array}$ & -2.9 & $\begin{array}{l}-3.9- \\
-1.7\end{array}$ & 0.00 & -0.7 & $-2.2-0.9$ & 0.45 \\
\hline \multicolumn{11}{|l|}{ Nausea } \\
\hline Control & 45 & $\begin{array}{l}1.8 \\
(3.1)\end{array}$ & 33 & $\begin{array}{l}0.3 \\
(0.8)\end{array}$ & -1.6 & $\begin{array}{l}-2.6- \\
-0.6\end{array}$ & 0.00 & & & \\
\hline Intervention & 41 & $\begin{array}{l}1.8 \\
(2.8)\end{array}$ & 26 & $\begin{array}{l}0.5 \\
(1.9)\end{array}$ & -1.3 & $-2.4--0.2$ & 0.03 & 0.3 & $\begin{array}{l}-1.2- \\
1.9\end{array}$ & 0.67 \\
\hline \multicolumn{11}{|c|}{ Disturbed sleep } \\
\hline Control & 48 & $\begin{array}{l}3.7 \\
(3.4)\end{array}$ & 33 & $\begin{array}{l}2.2 \\
(2.9)\end{array}$ & -1.5 & $-2.7--0.4$ & 0.01 & & & \\
\hline Intervention & 39 & $\begin{array}{l}4.3 \\
(3.3)\end{array}$ & 28 & $\begin{array}{l}1.8 \\
(2.7)\end{array}$ & -2.6 & $-3.9--1.2$ & 0.00 & -1.0 & $-2.8-0.8$ & 0.26 \\
\hline \multicolumn{11}{|c|}{ Being distressed } \\
\hline Control & 48 & $\begin{array}{l}3.3 \\
(3.2)\end{array}$ & 31 & $\begin{array}{l}2.2 \\
(2.7)\end{array}$ & -1.5 & $-2.5--0.6$ & 0.00 & & & \\
\hline Intervention & 40 & $\begin{array}{l}3.2 \\
(3.1)\end{array}$ & 28 & $\begin{array}{l}1.6 \\
(2.5)\end{array}$ & -1.6 & $-2.7--0.6$ & 0.00 & -0.1 & $-1.6-1.3$ & 0.87 \\
\hline \multicolumn{11}{|c|}{ Shortness of breath } \\
\hline Control & 48 & $\begin{array}{l}1.6 \\
(2.2)\end{array}$ & 33 & $\begin{array}{l}0.9 \\
(1.8)\end{array}$ & -0.6 & $-1.4-0.2$ & 0.14 & & & \\
\hline Intervention & 40 & $\begin{array}{l}1.2 \\
(2.1)\end{array}$ & 28 & $\begin{array}{l}0.9 \\
(1.8)\end{array}$ & -0.4 & $-1.2-0.5$ & 0.40 & 0.2 & $-0.9-1.4$ & 0.69 \\
\hline \multicolumn{11}{|c|}{ Difficulty remembering } \\
\hline Control & 47 & $\begin{array}{l}1.5 \\
(2.3)\end{array}$ & 32 & $\begin{array}{l}0.9 \\
(1.4)\end{array}$ & -0.7 & $-1.5-0.0$ & 0.06 & & & \\
\hline Intervention & 39 & $\begin{array}{l}1.5 \\
(2.3)\end{array}$ & 28 & $\begin{array}{l}0.9 \\
(1.8)\end{array}$ & -0.5 & $-1.3-0.3$ & 0.22 & 0.2 & $-0.9-1.3$ & 0.71 \\
\hline \multicolumn{11}{|c|}{ Lack of appetite } \\
\hline Control & 45 & $\begin{array}{l}3.1 \\
(3.7)\end{array}$ & 33 & $\begin{array}{l}0.9 \\
(1.8)\end{array}$ & -2.2 & $-3.4--1.0$ & 0.00 & & & \\
\hline Intervention & 36 & $\begin{array}{l}2.2 \\
(2.7)\end{array}$ & 28 & $\begin{array}{l}1.0 \\
(2.5)\end{array}$ & -1.2 & $-2.5-0.1$ & 0.07 & 1.0 & $-0.7-2.8$ & 0.24 \\
\hline \multicolumn{11}{|c|}{ Drowsy (sleepy) } \\
\hline Control & 47 & $\begin{array}{l}4.2 \\
(3.2)\end{array}$ & 33 & $\begin{array}{l}2.0 \\
(2.4)\end{array}$ & -2.2 & $-3.2--1.2$ & 0.00 & & & \\
\hline Intervention & 41 & $\begin{array}{l}3.7 \\
(2.9)\end{array}$ & 28 & $\begin{array}{l}1.8 \\
(2.8)\end{array}$ & -2.0 & $-3.1--0.9$ & 0.00 & 0.2 & $-1.3-1.6$ & 0.84 \\
\hline \multicolumn{11}{|l|}{ Dry mouth } \\
\hline Control & 48 & 3.5 & 30 & 1.3 & -2.4 & $\begin{array}{l}-3.5--1.3 \\
\text { ge } 11 / 20\end{array}$ & 0.00 & & & \\
\hline
\end{tabular}


(3.1)

(1.6)

\begin{tabular}{|c|c|c|c|c|c|c|c|c|c|c|}
\hline Intervention & 42 & $\begin{array}{l}3.8 \\
(3.3)\end{array}$ & 28 & $\begin{array}{l}2.2 \\
(2.9)\end{array}$ & -1.7 & $-2.8--0.6$ & 0.00 & 0.7 & $-0.8-2.3$ & 0.36 \\
\hline \multicolumn{11}{|l|}{ Sadness } \\
\hline Control & 47 & $\begin{array}{l}3.4 \\
(3.1)\end{array}$ & 32 & $\begin{array}{l}1.9 \\
(2.7)\end{array}$ & -1.7 & $-2.7--0.6$ & 0.00 & & & \\
\hline Intervention & 40 & $\begin{array}{l}3.1 \\
(3.3)\end{array}$ & 27 & $\begin{array}{l}1.3 \\
(2.4)\end{array}$ & -1.8 & $-2.9--0.6$ & 0.00 & -0.1 & $-1.7-1.5$ & 0.92 \\
\hline \multicolumn{11}{|l|}{ Vomiting } \\
\hline Control & 47 & $\begin{array}{l}0.9 \\
(2.6)\end{array}$ & 33 & $\begin{array}{l}0.2 \\
(0.7)\end{array}$ & -0.8 & $-1.6-0.1$ & 0.06 & & & \\
\hline Intervention & 40 & $\begin{array}{l}0.4 \\
(1.5)\end{array}$ & 28 & $\begin{array}{l}0.4 \\
(1.5)\end{array}$ & -0.0 & $-0.9-0.9$ & 0.95 & 0.8 & $-0.4-2.0$ & 0.19 \\
\hline \multicolumn{11}{|c|}{ Numbness/tingling } \\
\hline Control & 48 & $\begin{array}{l}2.4 \\
(3.2)\end{array}$ & 33 & $\begin{array}{l}1.9 \\
(2.5)\end{array}$ & -0.6 & $-1.5-0.3$ & 0.21 & & & \\
\hline Intervention & 39 & $\begin{array}{l}1.7 \\
(2.4)\end{array}$ & 28 & $\begin{array}{l}1.7 \\
(2.1)\end{array}$ & -0.3 & $-1.3-0.8$ & 0.61 & 0.3 & $-1.1-1.7$ & 0.64 \\
\hline
\end{tabular}

Head and neck symptoms

Problem with mucus in mouth throat

Control $\quad 48 \quad 3.2(3.1) \quad 33$

Intervention $39 \quad 3.5(3.2) \quad 28$

Difficulty with swallowing/chewing

$\begin{array}{llllllll}\text { Control } & 39 & 4.9(3.8) & 32 & 1.8(2.4) & -3.2 & -4.6--1.8 & 0.00\end{array}$

$\begin{array}{llllllll}\text { Intervention } & 39 & 4.5(3.3) & 27 & 2.1(3.3) & -2.3 & -3.7--0.9 & 0.00\end{array}$

Coughing/choking

Contro

$1.4(2.5) \quad 33 \quad 0.8(1.7)$

$1.9(1.9) \quad-1.2$

$-2.4--0.1$

0.04

$1.6(2.4)-2.0$

$-3.3--0.8$

0.00

$-0.8$

$-2.5-0.9 \quad 0.35$

$\begin{array}{lllll}\text { Intervention } & 39 & 1.7(2.9) & 28 & 0.8(2.1)\end{array}$

$-0.7$

$-1.7-0.4$

0.21

Difficulty with voice/speech

$\begin{array}{lllll}\text { Control } \quad 41 & 3.2(3.2) & 30 & 2.7(2.6)\end{array}$

$\begin{array}{lll}-0.9 & -2.1-0.2 & 0.11\end{array}$

$-0.2$

$-1.8-1.3 \quad 0.75$

Intervention $\quad 40 \quad 3.9(3.3) \quad 26 \quad 1.2(1.6)$

$-0.7$

$-1.9-0.6 \quad 0.28$

Skin pain/burning/rash

$\begin{array}{lllll}\text { Control } & 45 & 0.4(1.1) & 32 & 0.4(1.2)\end{array}$

$-2$.

$-4.2--1.6$

0.00

$-2.2$

$-4.0--0.4 \quad 0.02$

Intervention $\quad 40 \quad 0.5(1.5) \quad 26 \quad 0.5(1.1)$

$-0.0$

$-0.6-0.6$

0.98

Constipation

$\begin{array}{lllll}\text { Control } & 43 & 1.6(2.9) & 32 & 0.3(0.6)\end{array}$

0.1

$-0.6-0.7$

0.87

0.1

$-0.8-0.9 \quad 0.89$

$\begin{array}{lllll}\text { Intervention } & 38 & 1.7(2.9) & 25 & 0.6(1.8)\end{array}$

$\begin{array}{lll}-1.3 & -2.4--0.2 & 0.02\end{array}$

$\begin{array}{lll}-1.1 & -1.4-1.9 & 0.08\end{array}$

0.2

$-1.4-1.9$

0.79 


\begin{tabular}{|c|c|c|c|c|c|c|c|c|c|c|c|}
\hline \multicolumn{12}{|c|}{ Problem with tasting food } \\
\hline Control & 38 & $1.2(2.8)$ & 30 & $1.5(2.3)$ & & -0.4 & $-0.7-1.4$ & 0.48 & & & \\
\hline Intervention & 31 & $1.8(2.9)$ & 25 & $1.8(2.6)$ & & -0.3 & $-1.4-0.8$ & 0.61 & -0.6 & $-2.2-0.9$ & 0.39 \\
\hline \multicolumn{12}{|c|}{ Mouth/throat sores } \\
\hline Control & 41 & $2.4(2.9)$ & 32 & $0.4(1.1)$ & & -2.0 & $-3.3--0.8$ & 0.00 & & & \\
\hline Intervention & 37 & $3.6(3.5)$ & 24 & $0.5(1.7)$ & & -3.1 & $-4.4--1.7$ & 0.00 & -1.0 & $-2.9-0.8$ & 0.26 \\
\hline \multicolumn{12}{|c|}{ Problem with teeth and gums } \\
\hline Control & 43 & $1.1(2.5)$ & 32 & $0.7(1.2)$ & & -0.4 & $-1.3-0.5$ & 0.41 & & & \\
\hline Intervention & 38 & $1.6(2.9)$ & 26 & $0.6(1.5)$ & & -1.0 & $-2.0--0.0$ & 0.04 & -0.6 & $-1.9-0.7$ & 0.34 \\
\hline \multicolumn{12}{|l|}{ Interference } \\
\hline \multicolumn{12}{|l|}{ Activity } \\
\hline Control & 46 & $4.3(3.2)$ & 31 & $3.0(2.6)$ & -1.3 & & $-2.6--0.1$ & 0.04 & & & \\
\hline Intervention & 38 & $4.5(3.3)$ & 25 & $2.5(2.8)$ & -2.0 & & $-3.5--0.6$ & 0.01 & -0.7 & $-2.6-1.2$ & 0.47 \\
\hline \multicolumn{12}{|l|}{ Mood } \\
\hline Control & 45 & $3.4(2.8)$ & 29 & $2.1(2.9)$ & -1.4 & & $-2.5--0.4$ & 0.01 & & & \\
\hline Intervention & 38 & $3.0(2.6)$ & 25 & $2.1(2.8)$ & -1.2 & & $-2.3-0.0$ & 0.05 & 0.3 & $-1.3-1.9$ & 0.72 \\
\hline \multicolumn{12}{|l|}{ Work } \\
\hline Control & 33 & $4.2(4.5)$ & 31 & $2.5(2.9)$ & -1.8 & & $-3.5--0.1$ & 0.04 & & & \\
\hline Intervention & 30 & $5.3(4.4)$ & 26 & $2.6(2.8)$ & -2.9 & & $-4.8--1.2$ & 0.00 & -1.2 & $-3.6-1.3$ & 0.36 \\
\hline \multicolumn{12}{|c|}{ Relations with others } \\
\hline Control & 46 & $2.0(2.8)$ & 31 & $1.4(2.1)$ & -0.6 & & $-1.7-0.5$ & 0.26 & & & \\
\hline Intervention & 37 & $2.2(3.0)$ & 26 & $1.8(2.8)$ & -0.6 & & $-1.8-0.6$ & 0.32 & -0.0 & $-1.6-1.6$ & 0.98 \\
\hline \multicolumn{12}{|l|}{ Walking } \\
\hline Control & 44 & $1.3(2.6)$ & 31 & $1.0(2.4)$ & -0.3 & & $-1.3-0.8$ & 0.62 & & & \\
\hline Intervention & 40 & $1.3(2.4)$ & 25 & $1.3(2.7)$ & -0.0 & & $-1.2-1.2$ & 0.98 & 0.3 & $-1.3-1.9$ & 0.73 \\
\hline \multicolumn{12}{|c|}{ Enjoyment of life } \\
\hline Control & 44 & $2.0(2.8)$ & 30 & $1.3(2.2)$ & -0.7 & & $-1.9-0.4$ & 0.21 & & & \\
\hline Intervention & 40 & $2.4(3.2)$ & 26 & $1.5(2.6)$ & -1.0 & & $-2.3-0.2$ & 0.11 & -0.3 & $-1.9-1.5$ & 0.76 \\
\hline
\end{tabular}

Mean scores and SD are based on raw data. Change scores and $\mathrm{Cl}$ are based on linear mixed model.

There was a notable difference between groups regarding referrals in the emotional domain, where more IG patients ( $\mathrm{n}=28$ ) were advised to contact a psychologist than CG $(n=7)$, or a counsellor (IG: $n=51$; CG: $n=25)$. There were in total 183 referrals in IG compared to 119 in CG (Table 4). 


\begin{tabular}{|c|c|c|c|c|c|c|c|c|c|c|c|}
\hline \multirow[b]{2}{*}{ Type of referral } & \multirow[b]{2}{*}{ Group } & \multicolumn{3}{|c|}{ Time point 1} & \multicolumn{3}{|c|}{ Time point 2} & \multicolumn{4}{|c|}{ Time point 3} \\
\hline & & $\begin{array}{l}\text { In } \\
\text { hospital } \\
\text { (n) }\end{array}$ & $\begin{array}{l}\text { Primary } \\
\text { sector } \\
\text { (n) }\end{array}$ & $\begin{array}{l}\text { Advised } \\
\text { about } \\
\text { (n) }\end{array}$ & $\begin{array}{l}\text { In } \\
\text { hospital } \\
\text { (n) }\end{array}$ & $\begin{array}{l}\text { Primary } \\
\text { sector } \\
\text { (n) }\end{array}$ & $\begin{array}{l}\text { Advised } \\
\text { about } \\
\text { (n) }\end{array}$ & $\begin{array}{l}\text { In } \\
\text { hospital } \\
\text { (n) }\end{array}$ & $\begin{array}{l}\text { Primary } \\
\text { sector } \\
\text { (n) }\end{array}$ & $\begin{array}{l}\text { Advised } \\
\text { about } \\
\text { (n) }\end{array}$ & $\begin{array}{l}\text { Total no } \\
\text { of } \\
\text { referrals }\end{array}$ \\
\hline \multirow{2}{*}{$\begin{array}{l}\text { Swallowing } \\
\text { Therapist }\end{array}$} & CG & 1 & 22 & 4 & 6 & 0 & 3 & 0 & 0 & 0 & 36 \\
\hline & IG & 2 & 18 & 4 & 1 & 2 & 5 & 1 & 0 & 3 & 36 \\
\hline Speech & CG & 0 & 2 & 2 & 0 & 7 & 1 & 0 & 5 & 3 & 20 \\
\hline Pathologist & IG & 0 & 5 & 9 & 0 & 10 & 6 & 0 & 3 & 3 & 36 \\
\hline \multirow[t]{2}{*}{ Physiotherapist } & CG & 0 & 3 & 0 & 0 & 2 & 0 & 0 & 0 & 0 & 5 \\
\hline & IG & 0 & 3 & 0 & 0 & 1 & 0 & 1 & 1 & 3 & 9 \\
\hline \multirow[t]{2}{*}{ Dietician } & CG & 0 & 0 & 0 & 1 & 0 & 0 & 0 & 0 & 0 & 1 \\
\hline & IG & 0 & 0 & 0 & 0 & 0 & 0 & 0 & 0 & 0 & 0 \\
\hline \multirow[t]{2}{*}{ Dentist } & CG & 0 & 0 & 0 & 0 & 0 & 3 & 0 & 1 & 0 & 4 \\
\hline & IG & 0 & 0 & 1 & 0 & 0 & 1 & 0 & 0 & 1 & 3 \\
\hline \multirow{2}{*}{$\begin{array}{l}\text { Dental } \\
\text { hygienist }\end{array}$} & CG & 0 & 0 & 0 & 0 & 0 & 0 & 0 & 0 & 0 & 0 \\
\hline & IG & 0 & 0 & 0 & 0 & 0 & 0 & 0 & 0 & 1 & 1 \\
\hline \multirow[t]{2}{*}{ Sexologist } & CG & 0 & 0 & 0 & 0 & 0 & 0 & 0 & 0 & 0 & 0 \\
\hline & IG & 0 & 0 & 0 & 0 & 0 & 1 & 0 & 0 & 0 & 1 \\
\hline \multirow[t]{2}{*}{ Psychologist } & CG & 0 & 0 & 4 & 0 & 0 & 0 & 0 & 0 & 3 & 7 \\
\hline & IG & 0 & 0 & 9 & 0 & 0 & 11 & 0 & 0 & 8 & 28 \\
\hline \multirow[t]{2}{*}{ Counsellor } & $\mathrm{CG}$ & 0 & 0 & 19 & 0 & 0 & 3 & 0 & 0 & 3 & 25 \\
\hline & IG & 0 & 1 & 28 & 0 & 0 & 10 & 0 & 0 & 12 & 51 \\
\hline \multirow{2}{*}{$\begin{array}{l}\text { Spiritual } \\
\text { counsellor }\end{array}$} & CG & 0 & 0 & 0 & 0 & 0 & 0 & 0 & 0 & 0 & 0 \\
\hline & IG & 0 & 0 & 0 & 0 & 0 & 0 & 0 & 0 & 2 & 2 \\
\hline \multirow{2}{*}{$\begin{array}{l}\text { Cancer } \\
\text { rehabilitation }\end{array}$} & CG & 0 & 2 & 6 & 0 & 4 & 5 & 0 & 1 & 1 & 19 \\
\hline & IG & 0 & 2 & 3 & 0 & 2 & 0 & 0 & 2 & 2 & 11 \\
\hline \multirow{2}{*}{$\begin{array}{l}\text { Oral } \\
\text { rehabilitation } \\
\text { team }\end{array}$} & CG & 0 & 0 & 0 & 2 & 0 & 0 & 0 & 0 & 0 & 2 \\
\hline & IG & 0 & 2 & 0 & 1 & 1 & 0 & 0 & 1 & 0 & 5 \\
\hline \multirow{2}{*}{$\begin{array}{l}\text { Nursing } \\
\text { rehabilitation } \\
\text { team }\end{array}$} & CG & 23 & 0 & 4 & 1 & 0 & 0 & 0 & 0 & 0 & 28 \\
\hline & IG & 32 & 0 & 5 & 2 & 0 & 1 & 0 & 0 & 0 & 40 \\
\hline \multirow{2}{*}{$\begin{array}{l}\text { Homecare } \\
\text { nurse }\end{array}$} & CG & 1 & 1 & 0 & 1 & 0 & 0 & 0 & 0 & 0 & 3 \\
\hline & IG & 0 & 3 & 1 & 0 & 1 & 0 & 0 & 0 & 0 & 5 \\
\hline \multirow[t]{2}{*}{ Welfare officer } & $\mathrm{CG}$ & 0 & 0 & 0 & 0 & 1 & 0 & 0 & 0 & 0 & 1 \\
\hline & IG & 0 & 0 & 0 & 0 & 0 & 0 & 0 & 0 & 0 & 0 \\
\hline \multirow{2}{*}{$\begin{array}{l}\text { Patient support } \\
\text { group }\end{array}$} & CG & 0 & 0 & 2 & 0 & 0 & 0 & 0 & 0 & 0 & 2 \\
\hline & IG & 0 & 0 & 4 & 0 & 0 & 7 & 1 & 0 & 0 & 12 \\
\hline $\begin{array}{l}\text { Patient } \\
\text { counsellor }\end{array}$ & CG & 0 & 2 & 1 & 0 & 1 & 0 & 0 & 0 & 0 & 4 \\
\hline \multicolumn{12}{|c|}{ 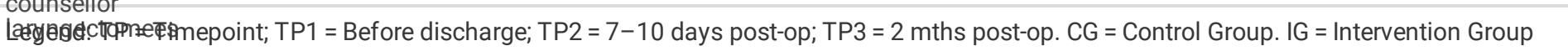 } \\
\hline
\end{tabular}




\begin{tabular}{|c|c|c|c|c|c|c|c|c|c|c|c|}
\hline & \multicolumn{4}{|c|}{ Time point 1} & \multicolumn{3}{|c|}{ Time point 2} & \multicolumn{4}{|c|}{ Time point 3} \\
\hline & IG & 0 & 2 & 0 & 0 & 1 & 0 & 0 & 0 & 1 & 4 \\
\hline \multirow{2}{*}{$\begin{array}{l}\text { Alcohol } \\
\text { cessation }\end{array}$} & CG & 0 & 0 & 2 & 0 & 0 & 1 & 0 & 0 & 0 & 3 \\
\hline & IG & 0 & 0 & 0 & 0 & 0 & 0 & 0 & 0 & 0 & 0 \\
\hline \multirow{2}{*}{$\begin{array}{l}\text { Smoking } \\
\text { cessation }\end{array}$} & CG & 0 & 1 & 14 & 0 & 3 & 8 & 0 & 0 & 6 & 32 \\
\hline & IG & 0 & 0 & 10 & 0 & 0 & 5 & 0 & 0 & 3 & 18 \\
\hline
\end{tabular}

Five most frequently chosen subjects on PCI by IG at time-point 1 were fear of cancer coming back (FCC) $(37.2 \%)$, swallowing $(27.9 \%)$, dental health/teeth (27.9\%), chewing/eating (27.9\%), and activity (27.9\%). Five most frequently chosen subjects for time-point 2 were: chewing/eating $(50 \%)$, cancer treatment (41.7\%), wound healing (33.3\%), FCC (33.3\%), dental health/teeth (33.3\%), and four most frequently chosen subjects at time-point 3 were: FCC (30.8\%), chewing/eating (23.1\%), dry mouth (19.2\%), and cancer treatment $(19.2 \%)$. Four other subjects were also chosen with a frequency of $15.4 \%$ : swallowing, sleeping, mood, and energy levels (Fig. 2).

$\mathrm{PCl}$ was used at time-point 1 by 43 , time-point 2 by 36 , and time-point 3 by 26 . No patients declined to use $\mathrm{PCl}$ at any time-point, but data for four patients were lost to follow-up due to IT-problems at time-point 3.

\section{Discussion}

To our knowledge, this is the first time PCl has been integrated in a nursing rehabilitation consultation, although it has been used routinely by individual oncologists or teams of oncologists and clinical nurse specialists in oncology clinics [34]. Nursing consultations using PROs in patients with cancer, or HNC specifically, have proven successful and aided in not only uncovering needs, but also facilitated discussion with the patient [12, 35].

This study was an RCT investigating the effect of applying an HNC-specific needs assessment tool, in clinical management and rehabilitation of patients with HNC early in the post-surgical period on HRQoL, and control of symptom burden. We did not find statistically significant differences between groups on GHS/QoL, and symptom burden. The study population already performed well in several areas post operatively, with only small improvements over time; however, there was a clinically relevant change for better in both groups over time on EORTC QLQ-H\&N35: GHS/QoL, emotional functioning, pain and appetite. Further, the magnitude of all symptoms on the MDASI-HN were mild and improving over time for both groups.

Other studies have shown that patients with HNC reported similar results to our study on GHS/QoL post-treatment [36, 37], while pain, appetite, swallowing, social eating, social contact, teeth and mouth opening all scored better ( $>10$-point difference) in our study [37]. Several studies have investigated the effect of a nurse-led intervention to improve HRQoL in patients with HNC [11, 12]. These have all had some effect on QoL long-term (6-24 months). The short-term symptom burden was highest in the early post-treatment period (4-10 weeks) with non-significant differences between groups [11]. A cohort study confirms that patients with HNC have a high symptom burden which peaks during treatment and need for symptom management during this period in the patient's trajectory [38]. This may indicate the need to look more in-depth at challenges surgically treated patients face in the short-term post-operative phase.

Using PCl during nurse consultations identified functional needs and concerns about cancer treatment and activity. Other studies on PCl in doctor's consultations have shown similar results [34]. Further, we found needs in emotional/existential areas, in particular the item FCC, as HNC cancer research has shown [39]. Fear of cancer recurrence (FOR) is common among patients with HNC [40]. Patients are reluctant to talk about emotional needs and FOR with HCPs [41] and are among those cancer patients most reluctant to ask for support [42], yet need support to talk about concerns around cancer recurrence [6]. As FCC was a highly chosen item on PCl, it could suggest patients need an opportunity or option for talking about existential or emotional needs and may be the reason more patients in IG were referred to emotional support. Thus, $\mathrm{PCl}$ could function as an avenue to address these subjects and support patient's in alleviating concerns or guide them to seek psychological or spiritual/existential assistance.

The $\mathrm{PCl}$ resulted in 64 (35\%) more referrals in IG compared to CG. Although our study did not attempt to measure the potential benefit of this, it is notable that more patients in IG were advised by the nurse to see a psychologist or counsellor for emotional support as a result of the chosen items. The majority of patients were advised based on the management manual to contact a counsellor at discharge, just as most patients were advised to contact a psychologist one to two weeks post-operatively. It is recognized that patients with HNC have complex needs, which can be 
addressed through multidisciplinary follow-up [6]. Since standard care does not require nurses to record identified needs, the number and type of referrals are assumed to indicate the nurse identified an unaddressed need in CG. Further, we do not know how many patients made use of these opportunities and thus benefitted from them. We believe this type of nursing rehabilitation consultation, can ensure patient preferences and priorities are incorporated in care aimed at improving HRQoL in patients with HNC.

\section{Strengths And Limitations}

Linguistic validation of the Danish version of $\mathrm{PCl}$ in cooperation with patients makes the future use of this instrument feasible in a larger Danish population of patients with HNC.

However, there are some limitations. Although $23 \%$ declined to participate, other studies have shown even higher rates. Kjaer et al. [43] found in a study of Danish patients with HNC less than $50 \%$ of the study population agreed to participate. Those who declined had a higher symptom burden, lower income, and less healthy lifestyle than participants. In this study, only a few patients (IG 11\%, CG 15\%) had a low educational background or were unemployed. Generally, the proportion of patients with HNC in Denmark and internationally with a low socioeconomic background is higher than other cancer populations $[44,45]$. As a result, it is possible that a high proportion of patients with middle to higher education in our study could explain why this group had lower symptom burden from the onset. The dropout and exclusion rate increased over time, as well as the amount of missing data, especially towards the end of the study. The latter may be due to patients independently completing the questionnaire at home seven days after their last visit to the out-patient department. Further, the final test time-point was approximately nine weeks after surgery, and patients may have needed more time to act on and benefit from consultations and advice given [12], particularly in terms of emotional and existential needs, to have an effect on HRQoL. Lastly, the multiple elements of the intervention may pose a risk when interpreting results. Future research should investigate whether nursing consultations using $\mathrm{PCl}$ in a larger study sample with a longer follow-up period, such as 6-24 months, could strengthen patient self-efficacy, reduce symptom burden, and improve HRQoL. Furthermore, qualitative research exploring patient experiences and satisfaction with nurse consultations using needs assessment tools may identify additional beneficial ways to employ them.

\section{Conclusion}

The study found applying an HNC needs assessment tool within nursing rehabilitation consultations over a nine-week period did not improve HRQoL or reduce symptom burden. However, the intervention suggests that important needs were identified and addressed, especially emotional and existential needs, which were accommodated through referrals and professional advice. In addition, nursing rehabilitation consultations using $\mathrm{PCl}$ can ensure that patient preferences and priorities are incorporated in their care.

\section{Declarations}

\section{Acknowledgements}

The authors would like to thank the study participants who contributed with their experiences. The rehabilitation team nurses, other HCPs who contributed to or supported this study, the project nurses I.M. Jelsdal, M. Sørensen, and A. K. Østergaard Madsen, as well as T. Bloch Jensen for leadership support.

\section{Funding}

This research was funded through two grants from Novo Nordisk Foundation (grant no: NNF160C0022338 and NNF180C0053227), and the Danish Nurses Organization (grant no: OTS/DSc).

\section{Conflict of interest}

The authors declare they have no conflict of interest.

\section{Availability of data and material}

The corresponding author has full control of all primary data and agrees to allow the journal to review their data if requested.

\section{Code availability}

Software application: R.

\section{Authors' contributions}

Annelise Mortensen: Conceptualization, Methodology, Formal analysis, Investigation, Resources, Writing - Original Draft, Project administration, Funding acquisition Irene Wessel: Conceptualization, Methodology, Formal analysis, Writing - Review \& Editing, Supervision Simon Rogers: 
Conceptualization, Methodology, Formal analysis, Writing - Review \& Editing, Supervision Anders Tolver. Formal analysis and investigation, Writing review and editing Mary Jarden: Conceptualization, Methodology, Formal analysis, Writing - Review \& Editing, Supervision, Funding acquisition.

\section{Compliance with ethical standards}

\section{Ethics approval}

The study was carried out in accordance with the Helsinki Declaration, Regional Ethics Committee of the Capitol Region of Denmark (16036032) and approved by Danish Data Protection Agency (2012-58-0004-05781).

\section{Consent to participate}

Informed consent was obtained from all participants included in the study.

\section{Consent to publish}

The participants provided informed consent regarding publishing data in this article.

\section{References}

[1] A. Mortensen and M. Jarden, "Early and late physical and psychosocial effects of primary surgery in patients with oral and oropharyngeal cancers: A systematic review," Oral Surg. Oral Med. Oral Pathol. Oral Radiol., vol. 121, no. 6, 2016.

[2] K. H. Nelke, W. Pawlak, H. Gerber, and J. Leszczyszyn, “Head and neck cancer patients' quality of life," Adv. Clin. Exp. Med., vol. 23, no. 6, pp. 1019-1027, 2014.

[3] J. Ringash, "Survivorship and quality of life in head and neck cancer," Journal of Clinical Oncology. 2015.

[4] N. T. A. Nguyen and J. Ringash, "Head and Neck Cancer Survivorship Care: A Review of the Current Guidelines and Remaining Unmet Needs," Curr. Treat. Options Oncol., vol. 19, no. 8, 2018.

[5] M. Giuliani, "Prevalence and nature of survivorship needs in patients with head and neck cancer," Head Neck, vol. July, pp. 1097-1103, 2016.

[6] J. Ringash et al., "Head and Neck Cancer Survivorship: Learning the Needs, Meeting the Needs," in Seminars in Radiation Oncology, 2018.

[7] B. a Murphy and J. Gilbert, "Dysphagia in head and neck cancer patients treated with radiation: assessment, sequelae, and rehabilitation.," Semin. Radiat. Oncol., vol. 19, no. 1, pp. 35-42, Jan. 2009.

[8] Danish Health Authority, Forløbsprogram for rehabilitering og palliation i forbindelse med kraeft (Guideline for rehabilitation and Palliation in Cancer). 2018.

[9] Capital Region of Denmark, "Rehabilitation and palliation - assessment of needs in patients with cancer," Guideline, 2018. [Online]. Available: https://vip.regionh.dk/vip/Admin/GUl.nsf/Desktop.html?Open. [Accessed: 28-Dec-2020].

[10] M. Larsson, B. Hedelin, and E. Athlin, "A supportive nursing care clinic: conceptions of patients with head and neck cancer., Eur. J. Oncol. Nurs., vol. 11, no. 1, pp. 49-59, Feb. 2007.

[11] E. Hansson, E. Carlström, L. E. Olsson, J. Nyman, and I. Koinberg, "Can a person-centred-care intervention improve health-related quality of life in patients with head and neck cancer? A randomized, controlled study," BMC Nurs., vol. 16, no. 1, pp. 1-12, 2017.

[12] I. C. Van Der Meulen et al., "Long-term effect of a nurse-led psychosocial intervention on health-related quality of life in patients with head and neck cancer: A randomised controlled trial," Br. J. Cancer, vol. 110, no. 3, pp. 593-601, 2014.

[13] H. Boyes, J. Barraclough, R. Ratansi, S. N. Rogers, and A. Kanatas, "Structured review of the patient-reported outcome instruments used in clinical trials in head and neck surgery," Br. J. Oral Maxillofac. Surg., vol. 56, no. 3, pp. 161-167, 2018.

[14] S. Rogers and B. Barber, "Using PROMs to guide patients and practitioners through the head and neck cancer journey," Patient Relat. Outcome Meas., vol. Volume 8, pp. 133-142, 2017.

[15] S. N. Rogers, D. Lowe, J. S. Brown, and E. D. Vaughan, "The University of Washington head and neck cancer measure as a predictor of outcome following primary surgery for oral cancer.," Head Neck, vol. 21, no. 5, pp. 394-401, Aug. 1999. 
[16] I. Bjelland, A. A. Dahl, T. T. Haug, and D. Neckelmann, "The validity of the Hospital Anxiety and Depression Scale," J. Psychosom. Res., vol. 52, no. 2, pp. 69-77, 2002.

[17] D. I. Rosenthal, "Measuring Head and Neck Cancer symptom burden: The development and validation of the M. D. Anderson symptom inventory, Head and Neck module," Head Neck, vol. 29, no. 10, pp. 923-931, 2007.

[18] C. Shunmugasundaram, C. Rutherford, P. N. Butow, P. Sundaresan, and H. M. Dhillon, “Content comparison of unmet needs self-report measures used in patients with head and neck cancer: A systematic review," Psychooncology., vol. 28, no. 12, pp. 2295-2306, 2019.

[19] S. N. Rogers, S. Ahiaku, and D. Lowe, "Is routine holistic assessment with a prompt list feasible during consultations after treatment for oral cancer?," Br. J. Oral Maxillofac. Surg., vol. 56, no. 1, pp. 24-28, 2018.

[20] P. A. Harris, R. Taylor, R. Thielke, J. Payne, N. Gonzalez, and J. G. Conde, "Research electronic data capture (REDCap)-A metadata-driven methodology and workflow process for providing translational research informatics support," J. Biomed. Inform., vol. 42, no. 2, pp. 377-381, 2009.

[21] K. Ribeiro and L. P. Kowalski, "APACHE II, POSSUM and ASA Scores and the Risk of Perioperative Complications in Patients With Oral or Oropharyngeal Cancer," Arch. Otolaryngol. Neck Surg., vol. 129, pp. 739-745, 2003.

[22] "Ministry of Finance," 2020. [Online]. Available: https://fm.dk/arbejdsomraader/kommuner-og-regioner/opgaver-for-kommuner-og-regioner/. [Accessed: 25-Jul-2020].

[23] S. N. Rogers, J. El-Sheikha, and D. Lowe, "The development of a Patients Concerns Inventory (PCI) to help reveal patients concerns in the head and neck clinic," Oral Oncol., vol. 45, no. 7, pp. 555-561, 2009.

[24] J. Young, A. Cund, M. Renshaw, A. Quigley, and A. Snowden, "Improving the care of cancer patients: holistic needs assessment," Br. J. Nurs., vol. 24, no. 4:S 17-20, 2015.

[25] S. N. Rogers, F. Thomson, and D. Lowe, "The Patient Concerns Inventory integrated as part of routine head and neck cancer follow-up consultations: Frequency, case-mix, and items initiated by the patient," Ann. R. Coll. Surg. Engl., vol. 100, no. 3, pp. 209-215, 2018.

[26] Dwamena and Fortin, Smith's Patient Centered Interviewing, 3rd ed. McGraw-Hill Education Medical, 2012.

[27] N. K. Aaronson, "The European Organisation for Research and Treatment of Cancer QLQ-C30: A quality-of-life instrument for use in international clinical trials in oncology," J. Natl. Cancer Inst., vol. 85, pp. 365-376, 1993.

[28] D. Osoba, G. Rodrigues, J. Myles, B. Zee, and J. Pater, "Interpreting the significance of changes in health-related quality-of- life scores," J. Clin. Oncol., vol. 16, no. 1, pp. 139-144, 1998.

[29] C. S. Cleeland, "Symptom burden: multiple symptoms and their impact as patient-reported outcomes.," J. Natl. Cancer Inst. Monogr., vol. 77030, no. 37 , pp. $16-21,2007$.

[30] C. S. Cleeland and T. R. Mendoza, Cancer Symptom Science. Measurement, Mechanisms and Management, 1st ed. New York: Cambridge University Press, 2011.

[31] M. Charlson, "A new method of classification of prognostic comorbidity for longitudinal studies: development and validation," J. Chronic Dis., vol. 40, no. 5, pp. 373-383, 1987.

[32] J. F. Carrillo, "The impact of treatment on quality of life of patients with head and neck cancer and its association with prognosis," J. Cancer Surg., vol. 42, no. 2016, pp. 1614-1621, 2016.

[33] R Core Team, "R: A language and environment for statistical computing." R Foundation for Statistical Computing, Vienna, 2013.

[34] S. N. Rogers, F. Thomson, and D. Lowe, "The Patient Concerns Inventory integrated as part of routine head and neck cancer follow-up consultations: Frequency, case-mix, and items initiated by the patient," Ann. R. Coll. Surg. Engl., vol. 100, no. 3, pp. 209-215, 2018.

[35] S. Duman-Lubberding et al., "Durable usage of patient-reported outcome measures in clinical practice to monitor health-related quality of life in head and neck cancer patients," Support. Care Cancer, vol. 25, no. 12, pp. 3775-3783, 2017.

[36] W. Derks, R. J. De Leeuw, G. J. Hordijk, and J. A. Winnubst, "Quality of life in elderly patients with head and neck cancer one year after diagnosis," Head Neck, vol. 26, no. 12, pp. 1045-1052, 2004.

[37] K. Bjordal et al., "A 12 country field study of the EORTC QLQ-C30 (version 3.0) and the head and neck cancer specific module (EORTC QLQH\&N35 ) in head and neck patients.," Eur. J. Cancer, vol. 36, pp. 1796-807, 2000. 
[38] C. O. Allen-Ayodabo et al., "Symptom burden among head and neck cancer patients in the first year after diagnosis: Association with primary treatment modality," Oral Oncol., vol. 99, no. June, p. 104434, 2019.

[39] a. Kanatas, N. Ghazali, D. Lowe, and S. N. Rogers, "The identification of mood and anxiety concerns using the patients concerns inventory following head and neck cancer," Int. J. Oral Maxillofac. Surg., vol. 41, pp. 429-436, 2012.

[40] N. Ghazali, E. Cadwallader, D. Lowe, G. Humphris, G. Ozakinci, and S. N. Rogers, "Fear of recurrence among head and neck cancer survivors: Longitudinal trends," Psychooncology., vol. 22, no. 4, 2013.

[41] J. Protheroe, H. Brooks, C. Chew-Graham, C. Gardner, and A. Rogers, '“Permission to participate?' A qualitative study of participation in patients from differing socio-economic backgrounds," J. Health Psychol., vol. 18, no. 8, pp. 1046-1055, 2013.

[42] S. Singer et al., "Predictors of emotional distress in patients with head and neck cancer," Head Neck, vol. February, pp. 180-187, 2012.

[43] T. Kjaer et al., "Do we reach the patients with the most problems? Baseline data from the WebCan study among survivors of head-and-neck cancer, Denmark," J. Cancer Surviv., vol. 10, no. 2, pp. 251-260, 2016.

[44] M. H. Olsen et al., "Socioeconomic position and stage at diagnosis of head and neck cancer - a nationwide study from DAHANCA.," Acta Oncol., vol. 54, no. 5, pp. 759-66, May 2015.

[45] D. M. Winn et al., "The INHANCE consortium: Toward a better understanding of the causes and mechanisms of head and neck cancer," Oral Dis., vol. 21, no. 6, pp. 685-693, 2015.

\section{Figures}

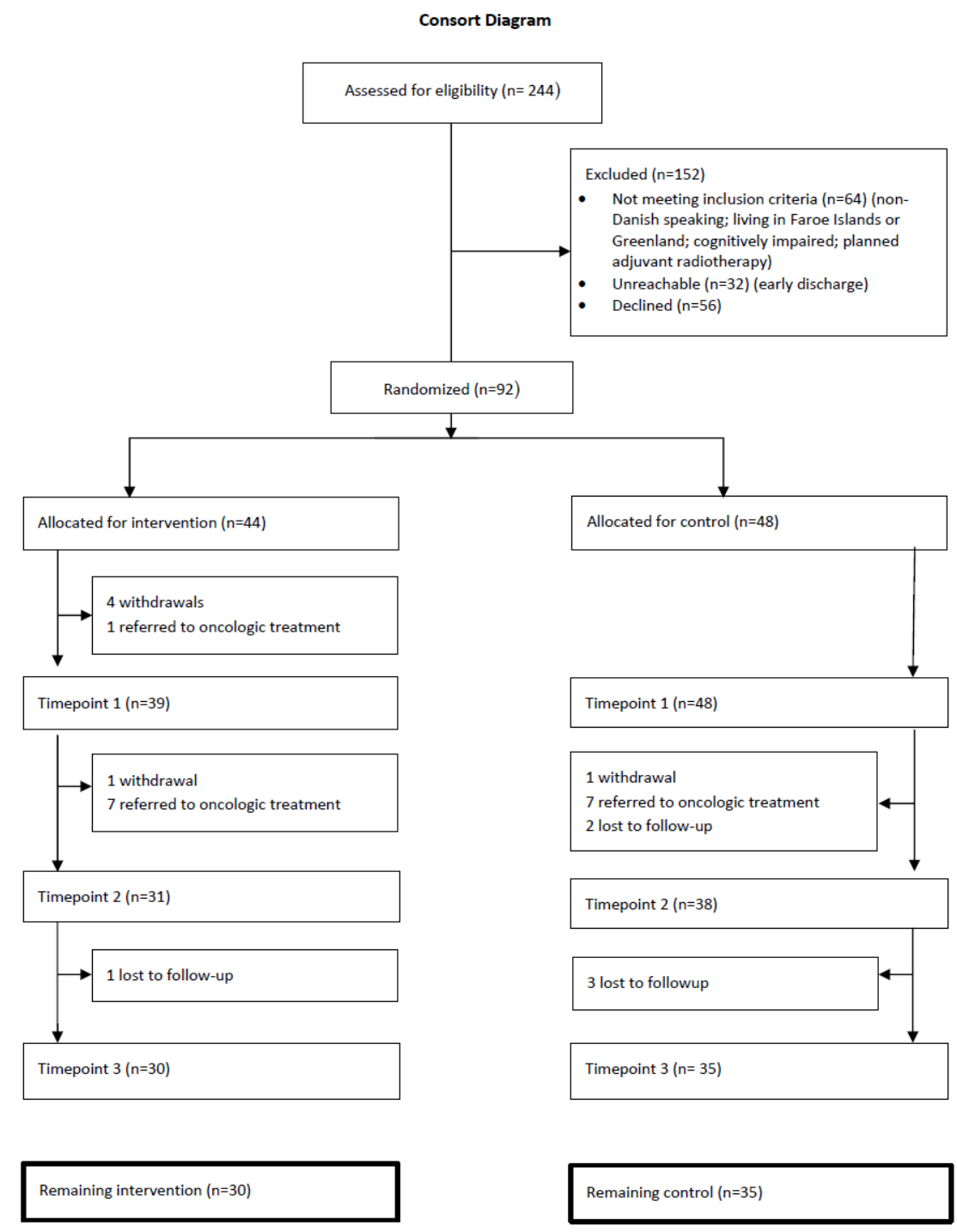


Figure 1

Consort Diagram

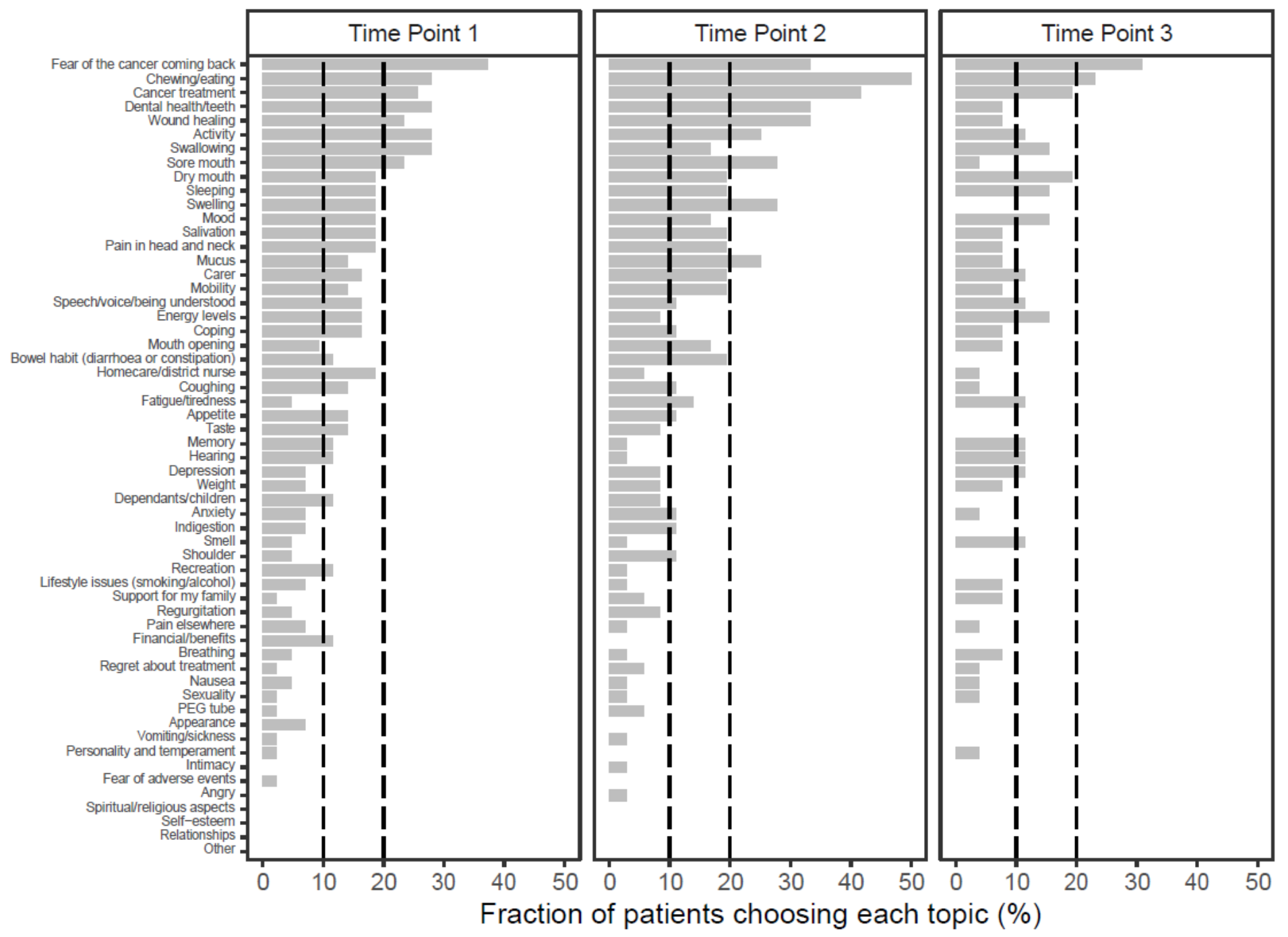

Figure 2

Five most frequently chosen subjects on PCI by IG at time-point 1 were fear of cancer coming back (FCC) (37.2\%), swallowing (27.9\%), dental health/teeth (27.9\%), chewing/eating (27.9\%), and activity (27.9\%). Five most frequently chosen subjects for time-point 2 were: chewing/eating (50\%), cancer treatment (41.7\%), wound healing (33.3\%), FCC (33.3\%), dental health/teeth (33.3\%), and four most frequently chosen subjects at time-point 3 were: FCC (30.8\%), chewing/eating (23.1\%), dry mouth (19.2\%), and cancer treatment (19.2\%). Four other subjects were also chosen with a frequency of $15.4 \%$ : swallowing, sleeping, mood, and energy levels 